\title{
Auditory system reorganization following cochlear implant in children with pre-lingual deafness.
}

\author{
Majid Karimi $^{1}$, Mohsen Ahadi ${ }^{*}$, Arash Bayat ${ }^{2,3}$, Nader Saki ${ }^{3}$ \\ ${ }^{1}$ Department of Audiology, Rehabilitation Research Center, School of Rehabilitation Sciences, Iran University of \\ Medical Sciences, Tehran, Iran \\ ${ }^{2}$ Musculoskletal Rehabilitation Research Center, School of Rehabilitation, Ahvaz Jundishapur University of Medical \\ Sciences, Ahvaz, Iran \\ ${ }^{3}$ Hearing Research Center, Imam Khomeini Hospital, Ahvaz Jundishapur University of Medical Sciences, Ahvaz, Iran
}

\begin{abstract}
Objective: To evaluate the auditory system reorganization following Cochlear Implant (CI) in prelingually deaf children using Cortical Auditory Evoked Potentials (CAEPs).

Methods: This is an open label prospective longitudinal study conducted on prelingually deaf Persian children ( $(\mathrm{n}=20)$ who underwent $\mathrm{CI}$ in a $\mathrm{CI}$ center in Khuzestan, Iran during 2015 to 2016. The subjects (10 boys, 10 girls; mean age: $2.48 \pm 1.05$ y) with profound pre-lingual hearing loss participated in this study. The CAEPs in response to speech stimuli $(/ \mathrm{m} /, / \mathrm{g} /$, and $/ \mathrm{t} /)$ were recorded using a HEARLab system. In all subjects the P1 latency of CAEPs was comparatively measured at three intervals: The baseline before $\mathrm{CI}$ and at two follow up points at 3- and 6-month post CI.

Results: The mean P1 values decreased with increasing time of implant use during the follow-up periods. The averaged P1 latency showed no significant difference between the different speech stimuli in all assessment intervals.

Conclusion: Our findings showed that $\mathrm{CI}$ influences the auditory system which can be measured in CAEPs.
\end{abstract}

Keywords: Cochlear implantation, Cortical auditory evoked potentials, Children.

Accepted on October 17, 2017

\section{Introduction}

Sensory deprivation caused by hearing loss leads to severe impairment of speech and language development in children. However, the effect of sensory deprivation can be recovered by the provision of sensory stimulation, such as sounds presented by Cochlear Implantation (CI) in children with a profound or severe-to-profound degree of hearing impairment [1-3]. Several studies have demonstrated that early CI in pre-lingual deaf children promotes their auditory function, speech perception, and linguistic development across time [4-6]. To evaluate the development of auditory and language skills, specific behavioral or objective protocols can be used and these protocols should be selected based on the child's age and developmental level. It has been shown that behavioral responses following $\mathrm{CI}$ activation are not always accurate and fast and can be affected by different factors including auditory deprivation duration, type of hearing loss, acquisition time (pre- or post-lingual), etiology of deafness and degree of residual hearing [7]. Considering the subjective entity of behavioral assessments and their limitations, objective evaluations with Cortical Auditory Evoked Potentials (CAEPs) have recently emerged as a beneficial method to objectively evaluate the benefits provided by $\mathrm{CI}$ in conjunction with behavioral measurements [8-10]. CAEPs can provide a window into the development and plasticity of central auditory pathways by recording the electrical activity of auditory cortex as an evoked response to a stimulating sound [11-14]. During young childhood, a large positive peak (P1) that originates from the auditory cortices dominates the CAEP [12] which is a reflective of the improvements in neural conduction time and synaptic strengthening improvements that occur in typical neural maturation. Therefore, recording and monitoring CAEPs may be a valuable approach for monitoring the cortical maturation in hearing-impaired children who underwent $\mathrm{CI}$. The present study aimed to investigate maturation and reorganization of the cortical auditory system following CI in prelingually deaf children using CAEPs in a major CI center in South Western of Iran. 


\section{Materials and Methods}

\section{Study population}

A total of 20 children (10 boys, 10 girls) with bilateral profound Sensorineural Hearing Loss (SNHL) participated in this open label prospective longitudinal study. All participants were born to hearing parents and have been identified as having SNHL within the 6 months of age their life. The subjects were selected from the implementation of new-born hearing screening program in South-western of Iran [14]. The subjects underwent CI surgery during 2015 to 2016, and the mean age of the subjects at the time of implantation was $2.48 \mathrm{y}$ $(\mathrm{SD}=1.05)$. All children had a complete insertion of electrode into the cochlea and have attended regular pre- and postoperative rehabilitation sessions.

The protocol and procedures of this study were approved by the Ethics Committee of the Iran University of Medical Sciences (No: 1395.9411301005) which were in complete accordance with the ethical standards and regulations of human studies of the Helsinki declaration (2014). After the enrolment of the subjects, all of the procedures of this study, potential benefits, and risks of this study were clearly explained to the parents of the children and after that the written consent forms were obtained from all the parents.

\section{Experimental procedures}

All CAEP assessments were performed in a sound-treated room using a HEARLab system (Frye Electronics, USA). Prior to the CAEP assessments, the subject's skin at the electrode placements was properly cleaned and prepared and specific recording gel was applied according to the instructions for recording of physiological evoked potentials. The test electrodes were positioned as follows: Active electrode on vertex $(\mathrm{Cz})$, Reference electrode on right or left mastoid (M1 and M2), and Ground electrode on forehead (Fz). The maximum inter-electrode's impedances were kept below $5 \mathrm{k} \Omega$. The children were evaluated while being alert, comfortably sit at directly in front of and one meter away the loudspeaker. The stimuli on the HEARLab system $(/ \mathrm{m} /, / \mathrm{g} /$, and $/ \mathrm{t} /)$ were extracted from running speech and presented at $65 \mathrm{~dB}$ SPL (alternate polarity; 200 epochs). The loudspeaker was placed directly in front of and 1 meter away from the sitting infant. The latency of P1wave was measured and compared at three time points: one as baseline signal measured prior to $\mathrm{CI}$ and two follow up points at 3-and 6-month intervals post-CI.

\section{Statistical analysis}

The data collected were analysed using statistical package of SPSS (Windows, version 19). Descriptive analyses were carried out using the mean and standard deviation. The normality of the data was confirmed by Kolmogorov-Smirnov test and then the comparisons were performed using parametric tests. Analysis of Variance (ANOVA) test was used to compare P1 latency across different time-points. The significance level was set as 0.05 for all statistical analyses.

\section{Results}

The mean values of P1 latency for different speech stimuli at pre-implantation and follow-up periods are presented in Table 1. It has been revealed that the mean P1 values decreased with increasing time of implant use during the follow-up stages. The repeated measure ANOVA showed a significant main effect of "Time" factor $(p<0.001)$. Pairwise comparisons on the significant main effect of "Time" showed a significant decrement in P1 latencies from pre-CI condition to "3-month" and "6-month" post-CI stages for all tested sounds $(\mathrm{p}<0.01)$.

Table 1. The mean PI latency ( $\pm S D$ ) before and after cochlear implantation.

\begin{tabular}{llll}
\hline & \multicolumn{3}{c}{ P1 latency (ms) } \\
\hline Stimulus Sound & Pre-Cl & 3 months after Cl & $\mathbf{6}$ months after CI \\
\hline$|\mathrm{m}|$ & $250.7 \pm 20.92$ & $190.0 \pm 21.59$ & $1566 \pm 19.34$ \\
\hline$|t|$ & $246.34 \pm 35.71$ & $171.6 \pm 45.61$ & $140.4 \pm 27.85$ \\
\hline$|\mathrm{g}|$ & $242.38 \pm 19.52$ & $158.8 \pm 26.51$ & $128.7 \pm 31.59$ \\
\hline
\end{tabular}

No significant differences in the group averages for P1 latency were found between the different speech stimuli of $/ \mathrm{m} /, / \mathrm{g} /$, and $/ \mathrm{t} /$.

\section{Discussion}

The present study investigated the maturation and reorganization status of the cortical auditory system following $\mathrm{CI}$ in prelingually deaf children using CAEPs in a major $\mathrm{CI}$ center in South-western of Iran. Congenital hearing loss leads to atypical organization of the Central Auditory Nervous System (CANS). However, the extent to which CANS pathways reorganize during sensory deprivation period is not yet fully understood. In recent years, CAEP responses have been applied clinically for the assessment of hearing aid and cochlear implant fittings. It has been suggested that the CAEPs provide information about the maturation of the auditory system and the effects of plasticity $[15,16]$. The early component of the CAEP waveform, $\mathrm{P} 1$, is generated by the auditory cortical and thalamic circuits of the CANS and exhibits reduced latency and increased amplitude into adolescence. This systematic decrease in latency has allowed the $\mathrm{P} 1$ to be extensively used as a biomarker for maturation of the CANS [17]. In particular, the P1 biomarker has been used for hearing-impaired children who use amplification devices (such as CI or hearing aid use) as an indicator of the effectiveness of interventions. The present study indicated that the mean P1 latencies decrease with increasing time of implant use during the follow-up time-points. This finding is in accordance with the findings of the Dorman et al. [11] who assessed the P1 latency in congenitally deaf children who had received CI following various periods of auditory deprivation. They reported that if children experienced less than $3.5 \mathrm{y}$ of sensory deprivation before CI operation, their P1 latencies following administration of 3-6 months of electrical stimulation fall into the range of normal children. 
During this study, children less than 3.5 y old underwent CI. It is known that the central auditory pathways are maximally plastic in response to auditory stimulation for a period of about $3.5 \mathrm{y}$ in early childhood. If stimulation is delivered within that period, then $\mathrm{P} 1$ latency and morphology reach age-normal values within 3-6 months following the onset of stimulation. Early diagnosis and intervention, while the central auditory system is highly plastic and degeneration has not been extensive, restoration of function may be possible with prosthesis such as the CI. It is known that the time length of auditory sensory deprivation that precedes the activation of cochlear implants considerably influences the redirecting of structures of central auditory pathways toward their primary function. This could explain the variability of the latency and amplitude of P1 wave and consequently the interference with speech perception. After the period of sensitivity, the benefits of $\mathrm{CI}$ in relation to speech perception tend to diminish with the advancing of an individual's age at the moment of activation. Moreover, hearing loss from birth up to the age of $3 \mathrm{y}$ significantly affect speech and language development of the children which consequently lead to several physical and cognitive impairments that negatively influence several aspects of life. Our findings support the results of the Van Dun study that showed no significant difference in the P1 latency between the different speech sounds of $/ \mathrm{m} /, / \mathrm{g} /$, and $/ \mathrm{t} /$ [15]. However, Golding et al. reported that the $/ \mathrm{t} /$ sound evoked cortical responses were significantly earlier in latency than for the $/ \mathrm{m} /$ and $/ \mathrm{g} /$ sounds [16-20].

\section{Conclusion}

The present study evaluated the effects of CI on the reorganization of the cortical auditory system in prelingually deaf children using CAEPs. The findings showed that the CI reduces the $\mathrm{P} 1$ latency where the reduction increases over. Moreover, the averaged P1 latency showed no significant difference between the different speech stimuli in different assessment intervals. Our findings demonstrated that $\mathrm{CI}$ influences the auditory system and CAEP is a reliable technique to measure these effects.

\section{Conflict of Interest Statement}

No potential conflict of interest relevant to this article was reported.

\section{Acknowledgements}

This study was supported by Iran University of Medical Sciences (Grant Number: 95-04-32-29805).

\section{References}

1. Leigh J. Communication development in children who receive a cochlear implant by 12 months of age. Otol Neurotol 2013; 34: 443-450.

2. Robinson K. Implications of developmental plasticity for the language acquisition of deaf children with cochlear implants. Int J Pediatr Otorhinolaryngol 1998; 46: 71-80.
3. Saki N, Bagheripour H, Bayat A, Saki Malehi A, Tavakoli M. Impact of duration of hearing loss on hearing performance of post-lingual cochlear implant users. Jundishapur Sci Med J 2017; 16: 153-160.

4. Ching TY. Early language outcomes of children with cochlear implants: Interim findings of the NAL study on longitudinal outcomes of children. Cochl Impl Int 2009; 10: 28-32.

5. Markman TM. Language development after cochlear implantation: an epigenetic model. J Neurodevelop Disord 2011; 3: 388.

6. Saki N,Yadollahpour A, Moniri S, Nikakhlagh S, Bayat A. Investigating the impacts of cochlear implantation on the happiness and self-esteem of mothers of children with severe hearing loss. Int J Mental Health Addict 2017; 15: 288-294.

7. Ouellet C, Cohen H. Speech and language development following cochlear implantation. J Neurolinguist 1999; 12: 271-288.

8. Sharma A, Dorman M, Spahr A, Todd NW. Early cochlear implantation in children allows normal development of central auditory pathways. Ann Otol Rhinol Laryngol 2002; 111: 38-41.

9. Eggermont JJ, Ponton CW, Don M, Waring MD, Kwong B. Maturational delays in cortical evoked potentials in cochlear implant users. Acta Oto-laryngologica 1997; 117: 161-163.

10. Visram AS, Innes-Brown H, El-Deredy W, McKay CM. Cortical auditory evoked potentials as an objective measure of behavioral thresholds in cochlear implant users. Hear Res 2015; 327: 35-42.

11. Dorman MF, Sharma A, Gilley P, Martin K, Roland P. Central auditory development: evidence from CAEP measurements in children fit with cochlear implants. J Commun Disord 2007; 40: 284-294.

12. Gilley PM, Sharma A, Dorman MF. Cortical reorganization in children with cochlear implants. Brain Res 2008; 1239: 56-65.

13. Gilley PM, Sharma A, Dorman M, Finley CC, Panch AS, Martin K. Minimization of cochlear implant stimulus artifact in cortical auditory evoked potentials. Clin Neurophysiol 2006; 117: 1772-1782.

14. Saki N, Bayat A, Nikakhlagh S, Hoseinabadi R. Universal new-born hearing screening in southwestern Iran. Int J Pediatr Otorhinolaryngol 2017; 97: 89-92.

15. Wunderlich JL, Cone-Wesson BK, Shepherd R. Maturation of the cortical auditory evoked potential in infants and young children. Hear Res 2006; 212: 185-202.

16. Sharma A, Gilley PM, Dorman MF, Baldwin R. Deprivation-induced cortical reorganization in children with cochlear implants. Int J Audiol 2007; 46: 494-499.

17. Sharma A, Glick H, Campbell J, Biever A. Central auditory development in children with hearing impairment: Clinical relevance of the P1 CAEP biomarker in children with multiple disabilities. Hear Bal Commun 2013; 11: 110-120. 
18. Sharma A, Dorman MF, Spahr AJ. Rapid development of cortical auditory evoked potentials after early cochlear implantation. Neuroreport 2002; 13: 1365-1368.

19. Golding M, Dillon H, Seymour J, Carter L. The detection of adult cortical auditory evoked potentials (CAEPs) using an automated statistic and visual detection. Taylor Francis 2009.

20. Van Dun B, Carter L, Dillon H. Sensitivity of cortical auditory evoked potential detection for hearing-impaired infants in response to short speech sounds. Audiol Res $2012 ; 2$.

\section{*Correspondence to}

Mohsen Ahadi

Department of Audiology

Rehabilitation Research Center

School of Rehabilitation Sciences

Iran 